\title{
NLRP3 Inhibitor Tranilast Attenuates Gestational Diabetes Mellitus in a Genetic Mouse Model
}

\author{
Jing $\mathrm{Cao}^{1} \cdot$ Qian Peng $^{2}$
}

Accepted: 10 January 2022 / Published online: 5 February 2022

(c) The Author(s) 2022

\begin{abstract}
Background and Objective This study was designed to explore the protective effects of a clinically available NLR family Pyrin domain-containing receptor 3 (NLRP3) inhibitor, tranilast, in gestational diabetes mellitus (GDM) mice.

Methods We used pregnant C57BL/KsJdb/+ $(\mathrm{db} /+)$ female mice as GDM mice, then orally administered $20 \mathrm{mg} / \mathrm{kg}$ of tranilast or metformin daily for 2 weeks. A glucose tolerance test and an insulin resistance test were used to evaluate the severity of diabetes in tranilast/metformin-treated GDM mice. After delivery, newborn mice were counted and weighed to measure their protective role on the reproductive outcome of GDM mice. Next, we determined the expression of NLRP3 and proinflammatory cytokines in the visceral adipose tissue and placenta of GDM mice using western blot and quantitative real-time-polymerase chain reaction. Furthermore, we determined the proinflammatory cytokines in the serum using an enzyme-linked immunosorbent assay.

Results Tranilast significantly ameliorated GDM symptoms, including maternal body weight, hyperglycemia, insulin insufficiency, glucose intolerance and insulin resistance, enlarged litter size, and reduced litter body weight. Additionally, tranilast remarkably reduced the elevated expression of NLRP3 and proinflammatory cytokines.

Conclusions Our data clarified the protective role of the NLRP3 inhibitor, tranilast, on GDM by inhibiting the activation of the NLRP3 inflammasome as well as inflammatory responses. The findings mean tranilast might serve as a therapeutic drug to treat GDM.
\end{abstract}

\section{Key Points}

Tranilast significantly ameliorated gestational diabetes mellitus symptoms, including maternal body weight, hyperglycemia, insulin insufficiency, glucose intolerance and insulin resistance, enlarged litter size, and reduced litter body weight.

Tranilast remarkably reduced the elevated expressions of the NLR family Pyrin domain-containing receptor 3 and proinflammatory cytokines.

Jing Cao

caoj_063aaa@163.com

1 Department of Endocrinology, Tianjin First Central Hospital, No. 24 Fukang Road, Nankai District, Tianjin 300192, China

2 School of Medicine, Nankai University, Nankai District, Tianjin, China

\section{Introduction}

Gestational diabetes mellitus (GDM) is diabetes that only occurs or is first diagnosed during pregnancy, leading to pre-eclampsia, premature rupture of fetal membrane, premature birth, and an increased risk of fetal malformations [1]. Currently, GDM comprises about $7 \%$ of all diabetes, and its incidence is gradually increasing owing to the growing pregnant age and rising standards of living [2]. Gestational diabetes mellitus exerts its effect on pregnant women, fetuses, and newborns, causing polyhydramnios and hypertension during pregnancy. Newborns are prone to metabolic abnormalities, such as respiratory distress syndrome, hypoglycemia, hypocalcemia, hypomagnesemia, polycythemia, and hyperbilirubinemia [3]. Furthermore, GDM significantly increases the risk of obesity and glucose intolerance in adolescence [4]. Additionally, GDM pregnancies and their offspring are also prone to developing type 2 diabetes mellitus in the future [4]. Although the diagnosis and therapeutic treatment of GDM have progressed in recent years, the pathogenesis of GDM remains 
largely unknown. However, growing evidence indicates that inflammation and inflammation-related mediators are closely associated with the occurrence of GDM $[5,6]$.

A previous publication discovered that the proportion of macrophages in the visceral adipose tissue (VAT) and placenta of patients with GDM was significantly higher than that of normal pregnancies [7,8], and these increased macrophages secreted proinflammatory factors such as tumor necrosis factor- $\alpha$ (TNF- $\alpha$ ) and interleukin- $1 \beta$ (IL$1 \beta$ ), which contributed to local and systemic inflammation, and eventually insulin resistance. As an important component of innate immunity, the NLR and pyrin domain containing receptor 3 (NLRP3) inflammasome plays an important role in the immune response. Because the NLRP3 inflammasome can be activated by the diversity of pathogens or danger signals, NLRP3 inflammasomes play a key role in a variety of diseases, such as familial periodic auto-inflammatory response and type 2 diabetes $[9,10]$. Current observations on the role of NLRP 3 in GDM suggest that NLRP3 is implicated as a therapeutic target of reproductive disorder, including GDM [11]; thus, the NLRP3 inhibitor might be a potential strategy to treat NLRP3-driven GDM. Several NLRP3 inhibitors have been recognized, including MCC950 and ODLT1177 [12, 13], and they were also reported to ameliorate some NLRP3 inflammasome-driven diseases.

Tranilast N-(3,4-dimethoxycinnamoyl) anthranilic acid (N-5) was first developed as an anti-allergic drug, then applied as an anti-inflammatory agent to treat inflammation-related diseases, such as atypical dermatitis, allergic conjunctivitis, keloids and hypertrophic scars, and bronchial asthma [14]. In recent years, its suppressive effect on inflammation was mostly due to its direct inhibition on NLRP3 inflammasomes [15]. Tranilast was further proved to regulate NLRP3 ubiquitination, which contributed to the degrading of NLRP3 [12]. Therefore, we aimed to explore the protective effect of the clinically available NLRP3 inhibitor tranilast [15] on GDM in a genetic GDM mouse model.

\section{Materials and Methods}

\subsection{GDM Mouse Model}

C57BL/KsJ+/+ (wild type, WT) and C57BL/KsJdb/+ $(\mathrm{db} /+)$ mice were purchased from Shanghai Model Organisms (Shanghai, China). Female $\mathrm{db} /+$ mice aged 8-12 weeks were mated with age-matched male WT mice, and mating was confirmed by the presence of a copulatory plug on the next day, which was designated as gestational day (GD) 0 . Pregnant $\mathrm{db} /+$ mice (GDM mice) were randomly divided into three groups, and orally treated with phosphate-buffered saline (control), $20 \mathrm{mg} / \mathrm{kg}$ of metformin (metformin), or 20 $\mathrm{mg} / \mathrm{kg}$ of tranilast (tranilast), respectively, from GD 0 daily for 2 weeks. The doses of metformin or tranilast were based on previous publications [16, 17]. Pregnant WT female mice, which were mated with WT male mice, were used as controls (WT). On GD 1, GD 8, and GD 15, pregnant mice from all groups were weighed. Non-fasting blood glucose levels were measured from the tail vein by a glucometer (Roche Diagnostics, Risch-Rotkreuz, Switzerland). Insulin levels in the serum were analyzed using the Ultra Sensitive Mouse Insulin ELISA kit according to the manufacturer's instructions (Alpco Diagnostics, Salem, NH, USA) [18]. After delivery, the litter size of offspring born from each pregnant mouse was recorded, and the body weight at birth of their offspring was weighed [19, 20]. The study was approved by the Ethics Committee of Tianjin First Central Hospital.

\subsection{Glucose and Insulin Tolerance Tests}

A glucose tolerance test and an insulin tolerance test were performed as previously described [21]. On GD 16, mice were intraperitoneally injected with $2 \mathrm{~g} / \mathrm{kg}$ of glucose after 6 hours of fasting, then blood glucose levels were measured at indicated timepoints $(0,30,60,90$, and 120 minutes after the glucose injection) using a glucometer. For insulin tolerance tests, mice were intraperitoneally injected with $1 \mathrm{mU} /$ $\mathrm{kg}$ of insulin after a 1-hour fast, then blood glucose levels were measured and recorded at indicated timepoints [22].

\subsection{Western Blot}

Visceral adipose tissues and placentas were collected from pregnant mice and digested in RIPA buffer supplemented with a protease inhibitor cocktail (Sigma-Aldrich, St Louis, MO, USA) to extract protein. Western blot was performed as previously described [23]. Briefly, the same amounts of protein were loaded into SDS-PAGE gel, and transferred onto a PVDF membrane. After blocking in 5\% milk, the membrane was incubated with a primary antibody followed by the incubation of a secondary antibody. The antibodies used were anti-NLRP3 (Santa Cruz Biotechnology, Inc., Santa Cruz, CA, USA, catalog: sc-134306), TNF- $\alpha$ (Abcam, Cambridge, MA, USA, catalog: ab6671), IL-6 (Abcam, catalog: ab208113), and $\beta$-actin (Sigma-Aldrich, clone number: AC-15). The protein bands were quantified using ImageJ software (National Institutes of Health, Bethesda, MD, USA) [24].

\subsection{Statistical Analysis}

Data were analyzed by one-way analyses of variance with a Tukey post-hoc test used for multiple comparisons, and a Student's $t$-test was used for two-group comparisons. Data 
are represented as means \pm standard deviations. $P<0.05$ was considered as a significant difference.

\section{Results}

\subsection{NLRP3 Inflammasomes were Activated in the VAT and Placenta of Mice with GDM}

We first explored the activation of NLRP3 inflammasomes in the VAT and placenta of GDM mice, where there were accumulated macrophages during pregnancy. In the VAT and placenta of WT pregnant mice, the NLRP3 protein level was slightly increased on GD 15 with no difference in the early stage of pregnancy (GD 1 and GD 8). Gestational diabetes mellitus mice had greatly elevated expressions of NLRP3 in the VAT (Fig. 1A-C) and placenta (Fig. 1D-F) on GD 8 , and its expression was further elevated on GD 15. These data indicated that NLRP3 inflammasomes were gradually activated in the VAT and placenta of GDM mice.

\subsection{Tranilast Attenuated the Symptoms of GDM in GDM Mice}

Next, GDM mice were orally administered $20 \mathrm{mg} / \mathrm{kg}$ of metformin, $20 \mathrm{mg} / \mathrm{kg}$ of NLRP3 inhibitor tranilast, or phosphate-buffered saline daily from GD 1 for 2 weeks as shown in Fig. 2A. Compared with WT mice, GDM mice in the control group gradually displayed GDM symptoms from GD 1 to GD 15, including elevated maternal body weight and blood glucose as well as decreased insulin levels (Fig. 2B-D). Metformin has proven to be a safe and effective drug to treat GDM [25]; therefore, we compared the efficacies of metformin and tranilast on GDM. Compared with GDM mice, metformin remarkably decreased the maternal body weight and blood glucose, and increased insulin levels, but all of these GDM symptoms could be further attenuated by treatment with tranilast. Furthermore, metformin ameliorated glucose and insulin intolerance in GDM mice, and tranilast further increased glucose and insulin sensitivity (Fig. 3A, B). Altogether, these data indicated that tranilast significantly attenuated the symptoms of GDM, and its therapeutic effect on GDM symptoms was even better than that of metformin.

\subsection{Tranilast Inhibited NLRP3 Expressions in the VAT and Placenta of GDM Mice}

To explore the role of the NLRP3 inhibitor on GDM, we first analyzed NLRP3 expressions in the VAT and placenta of mice with GDM. As expected, GDM mice displayed a higher expression of NLRP3 than WT mice,
Fig. 1 NLR family Pyrin domain-containing receptor 3 (NLRP3) inflammasome was activated in the visceral adipose tissue and placenta of mice with gestational diabetes mellitus (GDM). The visceral adipose tissue and placenta were isolated from wild-type mice and GDM mice on gestational day (GD) 1, GD 8, and GD 15 for examining the activation of the NLRP3 inflammasome. The expressions of NLPR3 in the visceral adipose tissue (A-C) and placenta (D-F) were detected via western blot. $P$-values for the analysis of variance in panels $\mathbf{B}, \mathbf{C}, \mathbf{E}$, and F were 0.0027, 0.0081, 0.0045, and 0.0019 , respectively

\section{A Visceral adipose tissue}

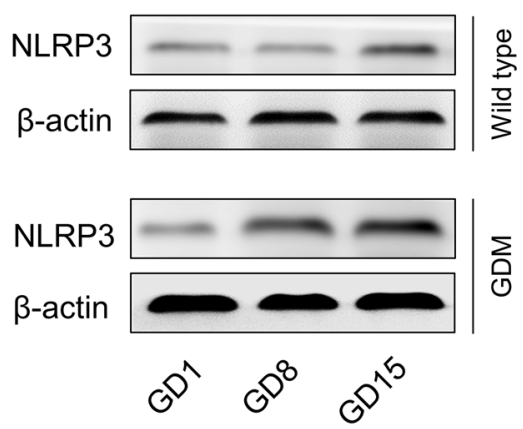

D

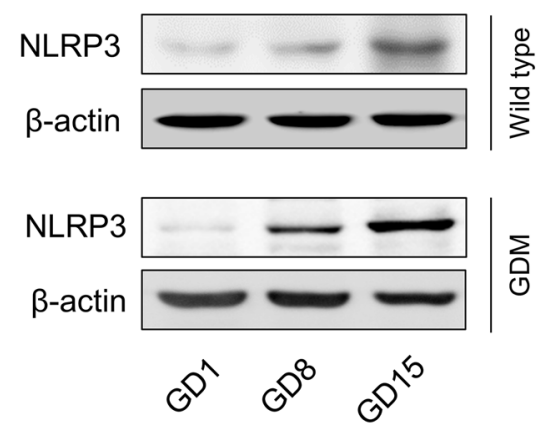

B

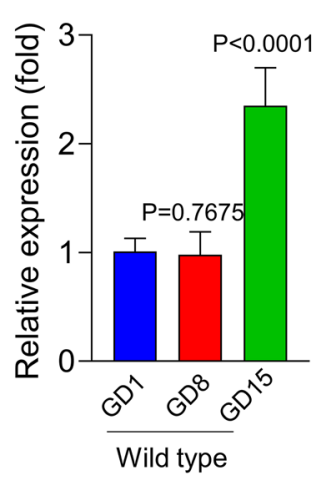

E

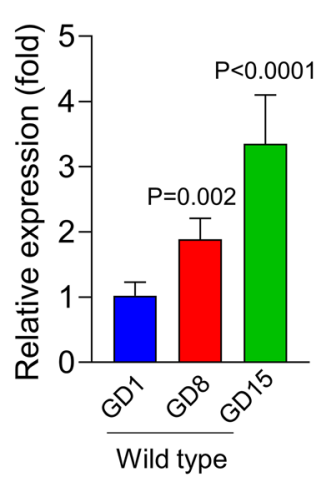

C

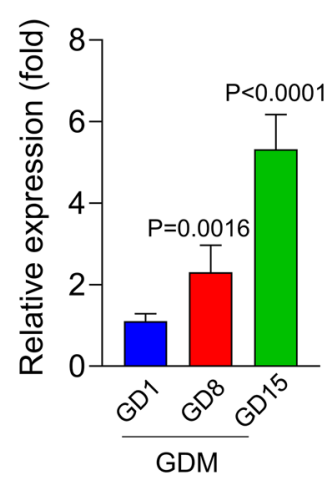

F

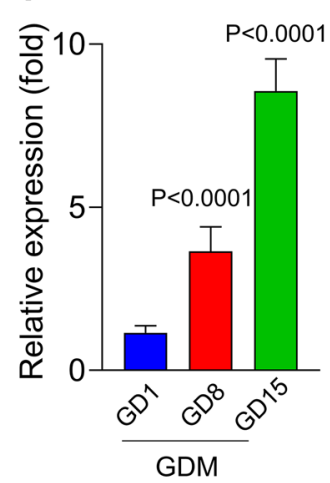




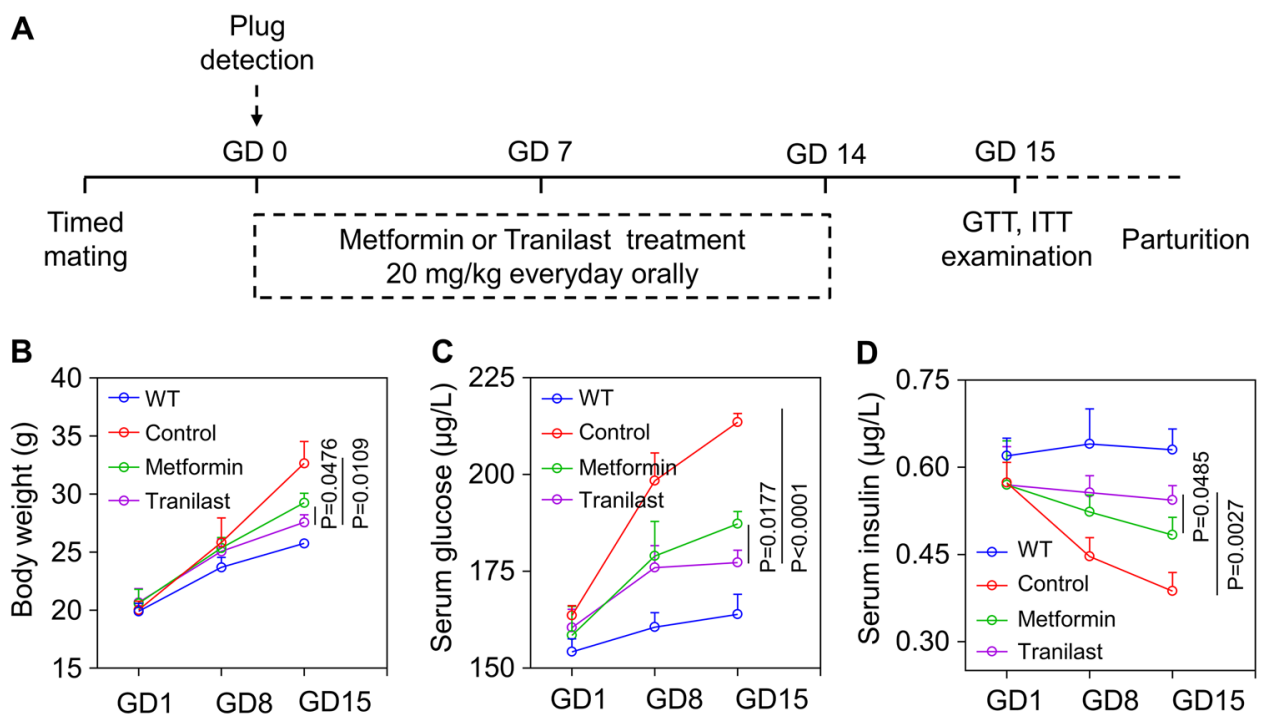

Fig. 2 Administration of the NLR family Pyrin domain-containing receptor 3 inhibitor tranilast attenuates gestational diabetes mellitus symptoms in mice. (A) Schematic of the experimental protocol. Maternal body weight, blood glucose level, and insulin level were recorded; a glucose tolerance test (GTT) and an insulin tolerance test (ITT) were performed. Maternal body weight (B), blood glucose level

which could be significantly restored by tranilast both in the VAT (Fig. 4A, C) and placenta (Fig. 4C, D). However, metformin had no effect on the increases in NLRP3 expression.

\subsection{Tranilast Inhibited Inflammation in GDM Mice}

Next, we evaluated the protective role of tranilast on inflammation in GDM mice. Inflammation activated in GDM mice, as reflected by the increased proteins

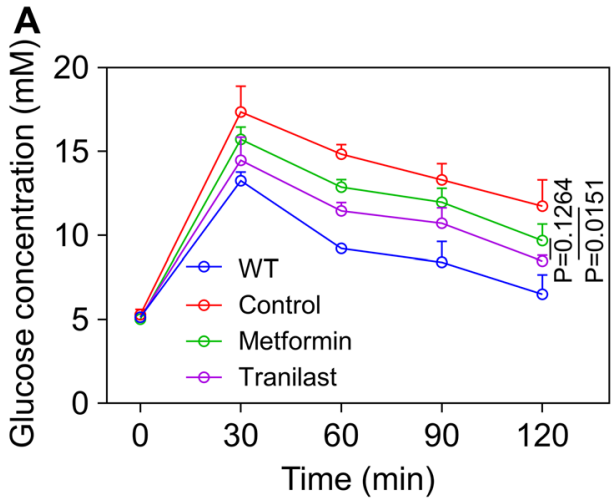

Fig. 3 Tranilast improved glucose and insulin sensitivity in gestational diabetes mellitus mice. (A) Effect of metformin or tranilast treatment on glucose tolerance in gestational diabetes mellitus mice. (B) Effect of metformin or tranilast treatment on insulin serum levels
(C), and insulin level (D) were measured on gestational day (GD) 1, 8 , and 15 from all groups. Data were presented as means \pm standard deviations. $n=8$. ${ }^{*} p<0.05,{ }^{* *} p<0.01$, $* * * p<0.001$, vs phosphate-buffered saline-treated gestational diabetes mellitus mice at GD 15. $P$-values for the analysis of variance in panels $\mathbf{B}, \mathbf{C}$, and $\mathbf{D}$ were $0.012,0.0014$, and 0.0482 , respectively. $W T$ wild type

and genes of proinflammatory cytokines, including TNF- $\alpha$ and IL- 6 , was reduced by metformin in the VAT (Fig. 5A-B and E) and placenta (Fig. 5C-D and F). Moreover, secreted TNF- $\alpha$ and IL- 6 in the serum, which were increased in GDM mice, were significantly decreased by tranilast or metformin (Fig. 5G-H). Notably, compared with metformin, tranilast further decreased the expressions of TNF- $\alpha$ and IL-6. Thus, tranilast significantly suppressed the inflammatory responses in GDM mice.

B

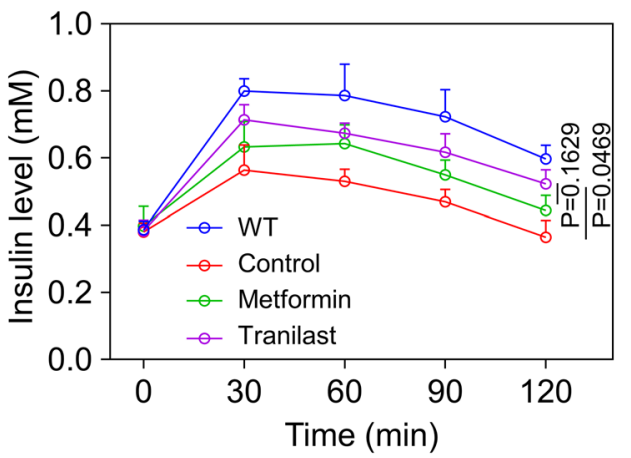

in gestational diabetes mellitus mice. Data were presented as means \pm standard deviations. $n=8$. $P$-values for the analysis of variance in panels A and B were 0.0445 and 0.0489 , respectively. WT wild type 
A

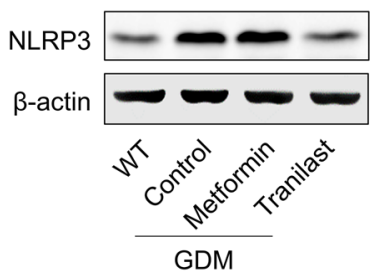

B

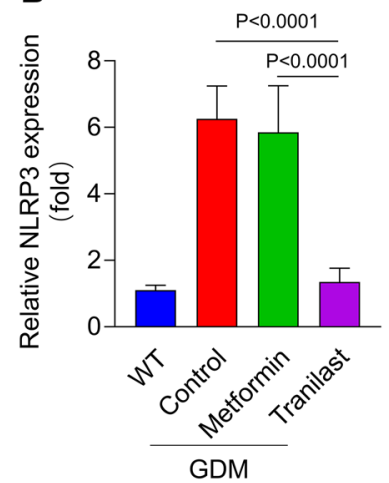

C

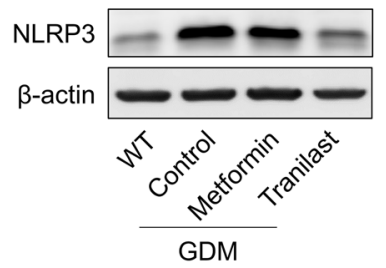

D

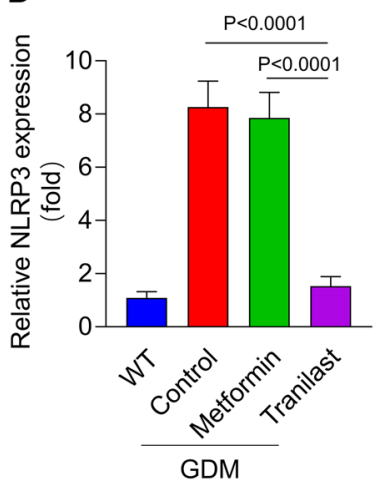

Fig. 4 Tranilast inhibited NLR family Pyrin domain-containing receptor 3 (NLRP3) expressions in the visceral adipose tissue and placenta of mice with GDM. Expressions of NLRP3 in the visceral adipose tissue (A) and (B) and placenta $(\mathbf{C}$ and $\mathbf{D})$ of pregnant wildtype (WT) mice or treated GDM mice (gestational day 16) were detected by western blot. $\beta$-Actin was set as the control protein. Data were presented as means \pm standard deviations. $n=8$. $* * * p<0.001$, vs phosphate-buffered saline-treated GDM mice. $P$-values for the analysis of variance in panels $\mathbf{B}$ and $\mathbf{D}$ were both $<0.0001$

\subsection{Tranilast Improved Reproductive Outcomes of Pregnant GDM Mice}

Next, we measured the effect of tranilast on the reproductive outcomes of pregnant mice. The offspring of GDM mice had a smaller litter size and heavier body weight than the offspring of WT mice. Tranilast significantly increased the litter size and decreased their body weight; however, metformin had no effect on the reproductive outcomes of GDM mice (Fig. 6A-B).

\section{Discussion}

Gestational diabetes mellitus is a hyperglycemic state during pregnancy that causes severe damage to both pregnant women and their offspring. Our study investigated the protective effect of the NLRP3 inhibitor, tranilast, against GDM, and explored the underlying mechanisms.

The pathophysiology of GDM is complex and its determination of risk factors is also complicated by inconsistent

diagnostic criteria, but obesity is a well-established and well-known risk factor of GDM. Inflammation is closely associated with obesity as well as GDM. Inflammation might also be the pathophysiological link between GDM and type 2 diabetes as well as cardiovascular diseases in patients with GDM after delivery [26, 27]. Therefore, based on the significant impact of systemic inflammation on the development of GDM, a variety of anti-inflammatory agents is always investigated as therapeutic candidate drugs for GDM [28].

Chronic inflammation is characterized as the excessive secretions of proinflammatory cytokines such as TNF- $\alpha$, IL-6, IL-1 $\beta$, C-reactive protein, and IL-18, which are the most common increased proinflammatory cytokines in the circulating system of patients with GMD. However, there are several sources of proinflammatory cytokines. Therefore, instead of measuring secretions of proinflammatory cytokines in the circulating blood, we evaluated the protein expressions of proinflammatory cytokines in the VAT and placenta. Our project explored the activation of the NLRP3 inflammasome in these two parts; therefore, protein levels of cytokines in the VAT and placenta were more representative of the inflammatory response than their secretions in the blood. In our study, we evaluated the expressions of TNF- $\alpha$ and IL- 6 as inflammatory markers to indicate the inflammatory levels, and our data clearly demonstrated that inflammation was activated in the VAT and placenta of genetic GDM mice, which was consistent with previous studies.

Previous studies indicated that macrophages were significantly accumulated in the VAT and placenta of patients with GDM [7, 8], which facilitated the secretions of proinflammatory cytokines as well as the activation of the NLRP3 inflammasome. That was the reason why we explored the VAT and placenta in our study. In addition, VAT and placenta were recognized as major sources of proinflammatory cytokines, and secreted cytokines could stimulate the production of adipokines, which in turn accelerated the inflammatory responses. Notably, emerging evidence discovered that a cross-talk between adipose tissue and the placenta played an important role during pregnancy [8]. This might explain the cross-talk between the VAT and placenta that facilitated the inflammatory responses to activate NLRP3 inflammasomes.

Emerging studies have demonstrated that the NLRP3 inflammasome is recognized as a novel target for several reproductive disorders, including pre-eclampsia, GDM, polycystic ovarian syndrome, preterm birth, and recurrent spontaneous abortion [12]. Based on the impact of the NLRP3 inflammasome on GDM, the NLRP3 inhibitor might be the candidate drug for treating GDM. However, until now, only Astragaloside IV was reported to effectively ameliorate GDM by inhibiting the NLRP3 inflammasome [29]. Therefore, our study is the first to prove that tranilast 
A

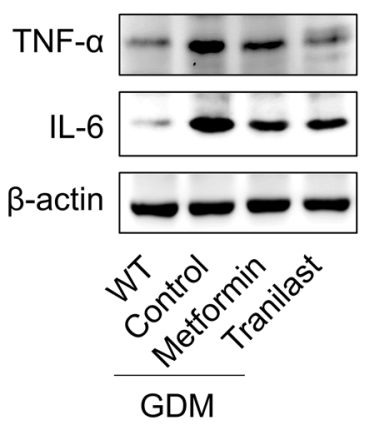

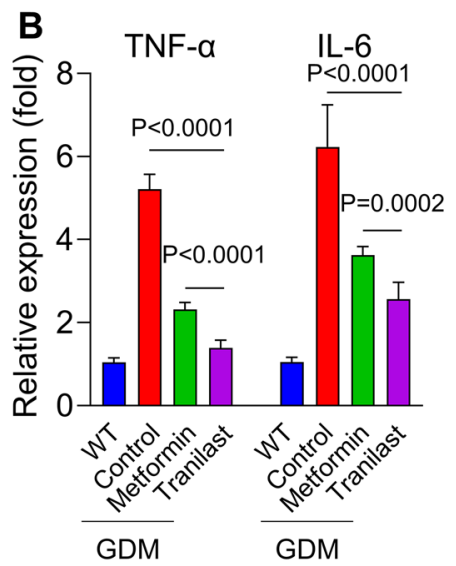
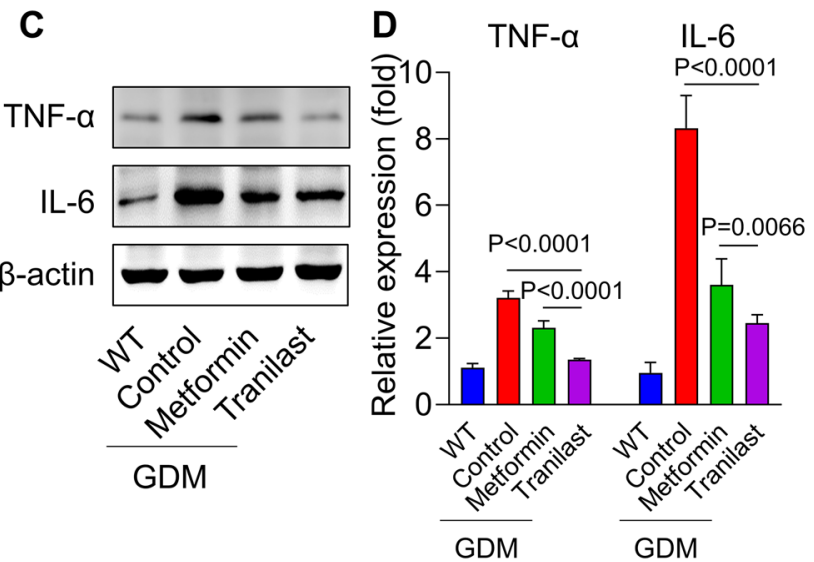
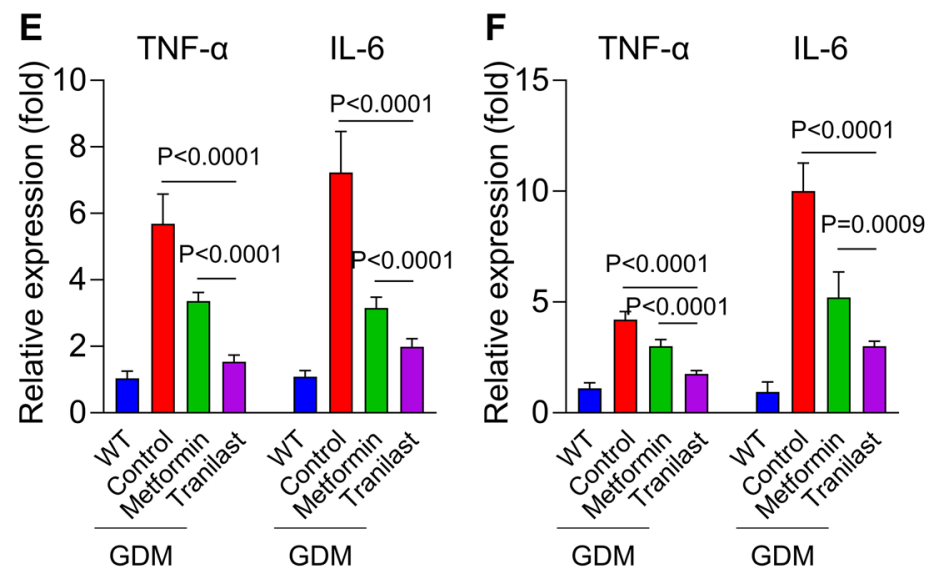

G

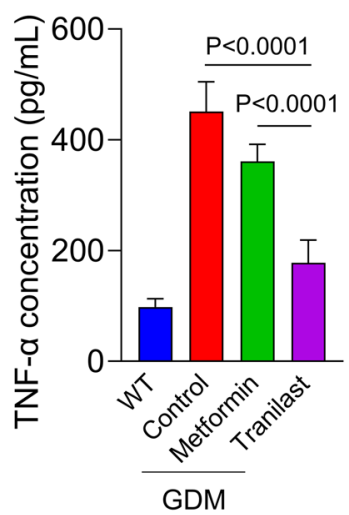

H

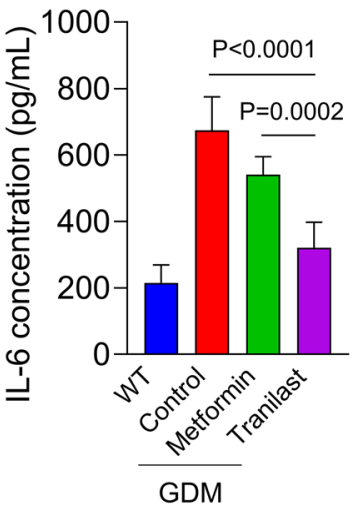

Fig. 5 Tranilast inhibited inflammation in gestational diabetes mellitus (GDM) mice. Expressions of interleukin-6 (IL-6) and tumor necrosis factor- $\alpha(\mathrm{TNF}-\alpha)$ in the visceral adipose tissue $(\mathbf{A}-\mathbf{B})$ and placenta $(\mathbf{C}-\mathbf{D})$ of pregnant wild-type (WT) mice or treated GDM mice were detected by western blot. $\beta$-Actin was set as the control protein. Messenger RNA levels of TNF- $\alpha$ and IL- 6 in the visceral adipose tissue $(\mathbf{E})$ and placenta $(\mathbf{F})$ of pregnant WT mice or treated
GDM mice were detected by real-time polymerase chain reaction. Serum levels of TNF- $\alpha(\mathbf{G})$ and IL6 (H) post-treatment were detected using an enzyme-linked immunosorbent assay. Data were presented as means \pm standard deviations. $n=8 . * p<0.05$, $* * p<0.01$, $* * * p$ $<0.001$, vs phosphate-buffered saline-treated GDM mice. $P$-values for the analysis of variance in panels $\mathbf{B}, \mathbf{D}, \mathbf{E}, \mathbf{F}, \mathbf{G}$, and $\mathbf{H}$ were all $<0.0001$

diabetes through a tumor growth factor- $\beta /$ Smad signaling pathway [30], and tranilast also attenuated mesenteric vascular collagen deposition and chymase-positive mast cells to improve diabetes [31], which might also be a potential mechanism to explain the protective effect of tranilast on GDM.

In addition, there are many publications exploring the relationship of NLRP3 in GDM, including activating the Toll-like receptor 4/MyD88/nuclear factor-kappa B signaling pathway [32], which suggests elucidating the mechanism of the protective role of tranilast on GDM should be explored in the future. Furthermore, the protective effect of tranilast in patients with GDM needs to be evaluated. We chose $20 \mathrm{mg} / \mathrm{kg}$ of tranilast as previously published [33], but to better evaluate its protective role in GDM, it might be better to try other doses of tranilast in the future. 

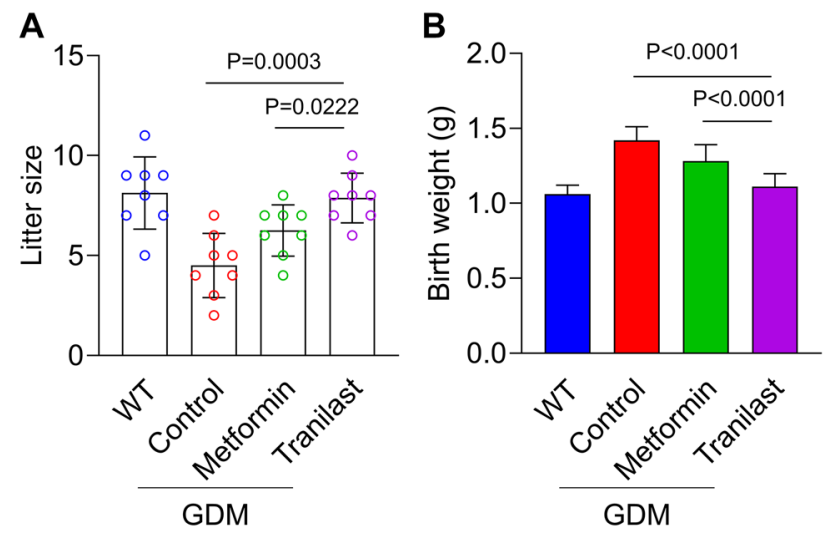

Fig. 6 Tranilast treatment improves reproductive outcomes of pregnant gestational diabetes mellitus (GDM) mice. Litter size (A) and body weight at birth (B) of offspring born by each female mouse from wild-type (WT) and $\alpha$ CCL2-treated GDM mice. Data represent means \pm standard deviations. $n=8$. $P$-values for the analysis of variance in panels $\mathbf{A}$ and $\mathbf{B}$ were 0.0022 and 0.0233 , respectively

\section{Conclusions}

Our data clearly clarify the protective role of tranilast on GDM by inhibiting the activation of the NLRP3 inflammasome as well as inflammatory responses in the VAT and placenta. Based on the findings, it is proposed that tranilast might serve as a therapeutic drug to treat GDM.

\section{Declarations}

Funding No sources of funding were received for the preparation of this article.

Conflicts of interest/competing interests The authors declare that they have no competing interests.

Ethics Approval The study was approved by the Ethics Committee of Tianjin First Central Hospital.

Consent to Participate Not applicable.

Consent for Publication Not applicable.

Availability of Data and Material Data may be obtained upon reasonable request to the authors.

\section{Code Availability None.}

Authors' Contributions Jing Cao and Qian Peng conducted the experiments, analyzed the data, and wrote the manuscript. Jing Cao conceived the study.

Open Access This article is licensed under a Creative Commons Attribution-NonCommercial 4.0 International License, which permits any non-commercial use, sharing, adaptation, distribution and reproduction in any medium or format, as long as you give appropriate credit to the original author(s) and the source, provide a link to the Creative
Commons licence, and indicate if changes were made. The images or other third party material in this article are included in the article's Creative Commons licence, unless indicated otherwise in a credit line to the material. If material is not included in the article's Creative Commons licence and your intended use is not permitted by statutory regulation or exceeds the permitted use, you will need to obtain permission directly from the copyright holder. To view a copy of this licence, visit http://creativecommons.org/licenses/by-nc/4.0/.

\section{References}

1. Szmuilowicz ED, Josefson JL, Metzger BE. Gestational diabetes mellitus. Endocrinol Metab Clin N Am. 2019;48:479-93. https:// doi.org/10.1016/j.ecl.2019.05.001.

2. Chiefari E, Arcidiacono B, Foti D, Brunetti A. Gestational diabetes mellitus: an updated overview. J Endocrinol Invest. 2017;40:899-909. https://doi.org/10.1007/s40618-016-0607-5.

3. Alfadhli EM. Gestational diabetes mellitus. Saudi Med J. 2015;36:399-406. https://doi.org/10.15537/smj.2015.4.10307.

4. Osgood ND, Dyck RF, Grassmann WK. The inter-and intragenerational impact of gestational diabetes on the epidemic of type 2 diabetes. Am J Public Health. 2011;101:173-9.

5. Pantham P, Aye IL, Powell TL. Inflammation in maternal obesity and gestational diabetes mellitus. Placenta. 2015;36:709-15. https://doi.org/10.1016/j.placenta.2015.04.006.

6. Nguyen-Ngo C, Jayabalan N, Salomon C, Lappas M. Molecular pathways disrupted by gestational diabetes mellitus. J Mol Endocrinol. 2019;63:R51-72. https://doi.org/10.1530/jme-18-0274.

7. Harlev A, Aricha-Tamir B, Shaco-Levy R, Tarnovscki T, Bashan $\mathrm{N}$, Rudich A, et al. Macrophage infiltration and stress-signaling in omental and subcutaneous adipose tissue in diabetic pregnancies. J Matern Fetal Neonatal Med. 2014;27:1189-94. https://doi.org/ 10.3109/14767058.2013.853734.

8. Jayabalan N, Nair S, Nuzhat Z, Rice GE, Zuñiga FA, Sobrevia $\mathrm{L}$, et al. Cross talk between adipose tissue and placenta in obese and gestational diabetes mellitus pregnancies via exosomes. Front Endocrinol (Lausanne). 2017;8:239. https://doi.org/10.3389/ fendo.2017.00239.

9. Shen HH, Yang YX, Meng X, Luo XY, Li XM, Shuai ZW, et al. NLRP3: a promising therapeutic target for autoimmune diseases. Autoimmun Rev. 2018;17:694-702. https://doi.org/10.1016/j. autrev.2018.01.020.

10. Zhang H, Luan S, Xiao X, Lin L, Zhao X, Liu X. Silenced microRNA-222 suppresses inflammatory response in gestational diabetes mellitus mice by promoting CXCR4. Life Sci. 2021;266: 118850. https://doi.org/10.1016/j.lfs.2020.118850.

11. Zhou F, Li C, Zhang S-Y. NLRP3 inflammasome: a new therapeutic target for high-risk reproductive disorders? Chin Med J. 2021;134:20.

12. Zahid A, Li B, Kombe AJK, Jin T, Tao J. Pharmacological inhibitors of the NLRP3 inflammasome. Front Immunol. 2019;10:2538. https://doi.org/10.3389/fimmu.2019.02538.

13. Lonnemann N, Hosseini S, Marchetti C, Skouras DB, Stefanoni $\mathrm{D}$, D'Alessandro A, et al. The NLRP3 inflammasome inhibitor OLT1177 rescues cognitive impairment in a mouse model of Alzheimer's disease. Proc Natl Acad Sci USA. 2020;117:32145-54. https://doi.org/10.1073/pnas.2009680117.

14. Darakhshan S, Pour AB. Tranilast: a review of its therapeutic applications. Pharmacol Res. 2015;91:15-28. https://doi.org/10. 1016/j.phrs.2014.10.009.

15. Huang Y, Jiang H, Chen Y, Wang X, Yang Y, Tao J, et al. Tranilast directly targets NLRP3 to treat inflammasome-driven diseases. 
EMBO Mol Med. 2018;10:e8089. https://doi.org/10.15252/ emmm.201708689.

16. Hemanth-Kumar P, Sarveswari SJPAC. A diversity-oriented concise synthesis of a new class bi, tri-podal quinoline derivatives with their in silico alpha-amylase and alpha-glucosidase binding studies. Polycycl Arom Compound. http://doi.org/10.1080/10406 638.2021.1996406

17. Han SY, Choi YHJP. Pharmacokinetic interaction between metformin and verapamil in rats: inhibition of the OCT2-mediated renal excretion of metformin by verapamil. Pharmaceutics. 2020;12:468. http://doi.org/10.3390/pharmaceutics12050468.

18. Yao L, Wan J, Li H, Ding J, Wang Y, Wang X, et al. Resveratrol relieves gestational diabetes mellitus in mice through activating AMPK. Reprod Biol Endocrinol. 2015;13:118. https://doi.org/10. 1186/s12958-015-0114-0.

19. Nguyen-Ngo C, Salomon C, Quak S, Lai A, Willcox JC, Lappas $M$. Nobiletin exerts anti-diabetic and anti-inflammatory effects in an in vitro human model and in vivo murine model of gestational diabetes. Clin Sci (Lond). 2020;134:571-92. https://doi.org/10. 1042/cs20191099.

20. Abdul Aziz SH, John CM, Mohamed Yusof NI, Nordin M, Ramasamy R, Adam A, et al. Animal model of gestational diabetes mellitus with pathophysiological resemblance to the human condition induced by multiple factors (nutritional, pharmacological, and stress) in rats. Biomed Res Int. 2016;2016:9704607. https://doi.org/10.1155/2016/9704607.

21. Liu Z, Iyer MR, Godlewski G, Jourdan T, Liu J, Coffey NJ, et al. Functional selectivity of a biased cannabinoid-1 receptor (CB1R) antagonist. ACS Pharmacol Transl Sci. 2021;4:1175-87.

22. Zou C, Zhang Q, Zhang S. Mogroside IIIE attenuates gestational diabetes mellitus through activating of AMPK signaling pathway in mice. J Pharmacol Sci. 2018;138:161-6. https://doi.org/10. 1016/j.jphs.2018.09.008

23. Liu Z, Luo H, Zhang L, Huang Y, Liu B, Ma K, et al. Hyperhomocysteinemia exaggerates adventitial inflammation and angiotensin II-induced abdominal aortic aneurysm in mice. Circ Res. 2012;111:1261-73. https://doi.org/10.1161/circresaha.112. 270520.

24. Toldo S, Marchetti C, Mauro AG, Chojnacki J, Mezzaroma E, Carbone S, et al. Inhibition of the NLRP3 inflammasome limits the inflammatory injury following myocardial ischemia-reperfusion in the mouse. Int J Cardiol. 2016;209:215-20. https://doi.org/ 10.1016/j.ijcard.2016.02.043.

25. Singh AK, Singh R. Metformin in gestational diabetes: an emerging contender. Indian J Endocrinol Metab. 2015;19:236-44. https://doi.org/10.4103/2230-8210.149317.

26. Skórzyńska-Dziduszko KE, Kimber-Trojnar Z, Patro-Małysza J, Olszewska A, Zaborowski T, Małecka-Massalska T. An interplay between obesity and inflammation in gestational diabetes mellitus. Curr Pharm Biotechnol. 2016;17:603-13.

27. Wolf M, Sauk J, Shah A, Smirnakis KV, Jimenez-Kimble R, Ecker JL, et al. Inflammation and glucose intolerance: a prospective study of gestational diabetes mellitus. Diabetes Care. 2004;27:21-7.

28. Lekva T, Norwitz ER, Aukrust P, Ueland T. Impact of systemic inflammation on the progression of gestational diabetes mellitus. Curr Diab Rep. 2016;16:26. https://doi.org/10.1007/ s11892-016-0715-9.

29. Zhang R, Zhang X, Xing B, Zhao J, Zhang P, Shi D, et al. Astragaloside IV attenuates gestational diabetes mellitus via targeting NLRP3 inflammasome in genetic mice. Endocrinology. 2019;17:1-8.

30. Li X, Liu J. Shang X Effect of tranilast on myocardial fibrosis in diabetes rats through TGF- $\beta /$ Smad pathway. Minerva Med. 2021;112:153-4. https://doi.org/10.23736/s0026-4806.19. 06178-0.

31. Jones SE, Gilbert RE, Kelly DJ. Tranilast reduces mesenteric vascular collagen deposition and chymase-positive mast cells in experimental diabetes. J Diabetes Compl. 2004;18:309-15. https://doi.org/10.1016/j.jdiacomp.2004.02.002.

32. Shen Z, Yang C, Zhu P, Tian C, Liang A. Protective effects of syringin against oxidative stress and inflammation in diabetic pregnant rats via TLR4/MyD88/NF- $\mathrm{BB}$ signaling pathway. Biomed Pharmacother. 2020;131:110681.

33. Choi JS, Huh BK, Lee SJ, Han M-J, Eom MR, Ahn H-J, et al. Tranilast-loaded tubular scaffold and surgical suture for suppression of stenosis after tracheal prosthesis transplantation. J Ind Eng Chem. 2020;82:81-8. 